\title{
Different stages of the evolution of cerebral aneurysms: joint analysis of mechanical test data and histological anal- ysis of aneurysm tissue
}

\author{
Daniil Parshin ${ }^{1,2, *}$, Anna Lipovka ${ }^{1,2}$, Andrey Dubovoy ${ }^{3}$, Mariya Vasilyeva ${ }^{1,4}$, Elena \\ Kuznetsova $^{4}$, and David Sergeevichev ${ }^{4}$ \\ ${ }^{1}$ Novosibitsk State University \\ ${ }^{2}$ Lavryentev Institute of Hydrodynamics \\ ${ }^{3}$ Federal Neurosurgical Center (Novosibirsk) \\ 4 "E. Meshalkin National medical research center" of the Ministry of Health of the Russian Federation
}

\begin{abstract}
In practical neurosurgery, an important issue is determining the status of the aneurysm and predicting its further growth, rupture or stabilization. The main approaches for the study of risk analysis asessment are computational hydrodynamics and analysis of the mechanics of the wall of cerebral aneurysm. In this paper, an analysis of various sections of the wall of cerebral aneurysm is given, combining mechanical test data and histological examination data. It was shown that, along with significant differences in mechanics, a different degree of calcification is observed in the tissue, which indicates a different level of impaired transport of substances inside the tissue.
\end{abstract}

\section{Introduction}

Cerebral aneurysms (CA) occur in 20-50 people per 1000 of population and depend a little on race and age $[1,2]$ while correlation with gender remains a controversial subject. One of the main challenges in modern neurosurgery is determining the stage of aneurysm for the subsequent estimating of the risks of its rupture.

The data of various mechanical experiments show significantly different results depending on the method of transportation of the samples, the sensitivity of the used tensile machine and the method of the experiment [3-7]. It was shown [8] that the difference in the mechanical properties of aneurysms among representatives and between samples with different experimental techniques can be comparable.

In this regard, the use of coupled tests for the classification of aneurysm tissues becomes important. In particular, an analysis of the relationship of the collagen fibers direction in a sample with its strength characteristics [9] using a confocal microscope is already used. A light scattering technique is used to determine the distribution of the main direction of the fibers and change this distribution during the mechanical load [10]. In the work [1] the histology of ruptured and unruptured aneurysms were compared, that showed the statistically different percentage of collagen, elastin, smooth muscle and endothelial cells.

Meanwhile, in many studies, a classification is made of ruptured and unruptured aneurysms

\footnotetext{
*e-mail: parshin@hydro.nsc.ru
} 
according to various criteria: maximum stress, maximum strain, distribution of the direction of collagen fibers, etc. All these results are indicated at different stages of development of aneurysm tissue, which, as shown, continues to develop for even several years [11]. Despite numerous studies in this area, it becomes clear that the main trigger destabilising the body of the aneurysm is still not elucidated.

These days the LIF method [12] is used to estimate the ratio of various proteins (nitrogenous bases), which are natural fluorophores, now is used for the study of cerebral aneurysms. In this paper, we conducted a comparative analysis of the results of a mechanical experiment with the data of the histological analysis of different areas of the aneurysm of the same patient. These areas (dome and neck) represent different stages of development of the same aneurysm, which is valuable experimental data. The ability to examine several tissue samples of the same aneurysm mechanically and histologically was obtained due to the large area of the body of the aneurysm and the developed over time method of its removal.

\begin{tabular}{|c|c|c|c|c|}
\hline Patient ID & Gender/Age & CA Status & CA localisation & Segment localisation \\
\hline T. & $\mathrm{M}(58)$ & Unruptured & MCA & CA neck \\
\hline T. & $\mathrm{M}(58)$ & Unruptured & MCA & CA dome \\
\hline
\end{tabular}

Table 1: Data on specimens. MCA-middle cerebral artery.

\section{Methods}

All specimens used in the experiment were obtained during the aneurysm clipping surgery in the Federal Neurological Center of Novosibirsk. After the extraction, the specimen is placed into saline of the temperature $+2-+5 \mathrm{C}$ and is delivered into the experimental laboratory under the same conditions. Overall time from the moment of specimen extraction up to the mechanical experiment does not exceed 24 hours.

The mechanical experiment is performed with the universal tensile machine Instron 5944, with specimen being in the biobath filled with solution heated to $37 \mathrm{C}$ during all the course of experiment. The specimen is fixed in the specially made clamps covered with sandpaper.

The mechanical experiment is performed in several stages with increasing engineering stress. After the test, some of the specimens were sent to histology analysis. On a rotary microtome, transverse histological sections $5 \mu \mathrm{m}$ thick were made. The preparations are stained with hematoxylin and eosin by the standard method (for review microscopy). Overall five specimens from three patients were studied in this work.

\section{Results}

\subsection{Mechanical test}

As a result of the cyclic uniaxial mechanical test with the samples, strain-stress diagrams are given in the Fig. 2 The values of the maximum stress and strain for each of the samples, which are listed in Table 2.

Below are the results of histological analysis of samples.

\subsection{Fragment T. (dome).}

The tissue is represented by a rather large fragment of an aneurysm-like altered artery wall. There are several pronounced subintimal calcification foci with disintegration in the center 


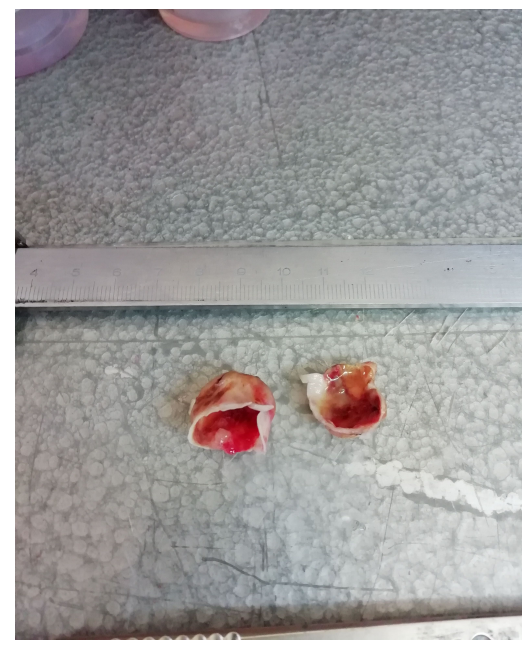

(a)

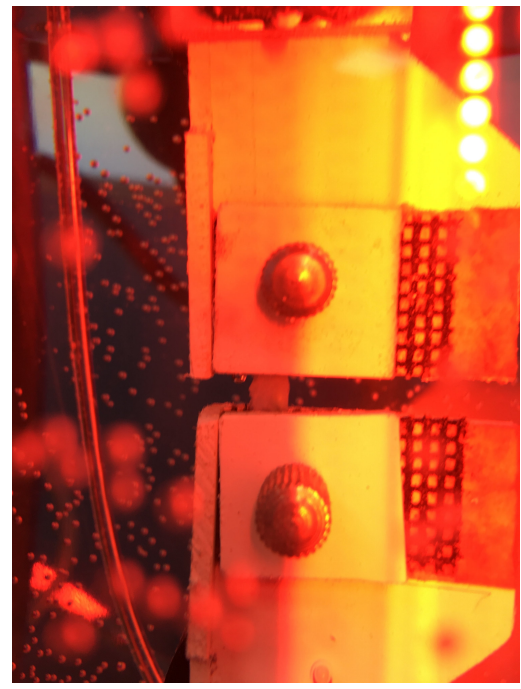

(b)

Figure 1: (a) - preparation of the specimens, (b) - specimen in the tensile machine.

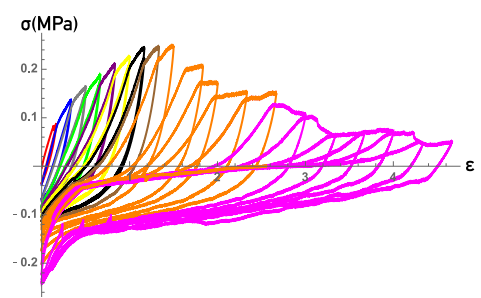

(a)

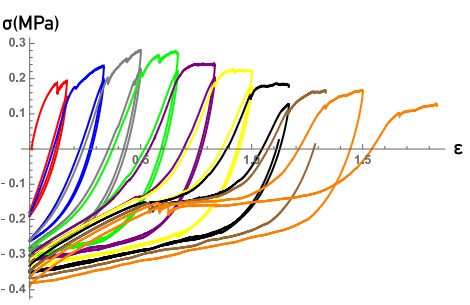

(b)

Figure 2: Strain-stress diagram: (a) - fragment of the dome, (b) - fragment of the neck.

of these zones. Around large foci, the presence of diffuse microcrystalline calcification is noted. The structure of the layers in the vessel wall is erased, the smooth muscle fibers of the middle layer are dystrophic altered, in several places they have completely lost their typical structure. There is total interfiber swelling of the vessel wall (Fig. 3a).

With high magnification, the presence of total microfragmentation of altered smooth muscle and connective tissue fibers is observed, with the result that in residual interfiber spaces, inclusions appear that are similar (similar) to atheromatous processes (Fig. 4b). That is also accompanied by the appearance of thin-walled blood vessels of small diameter (neovasculogenesis). 


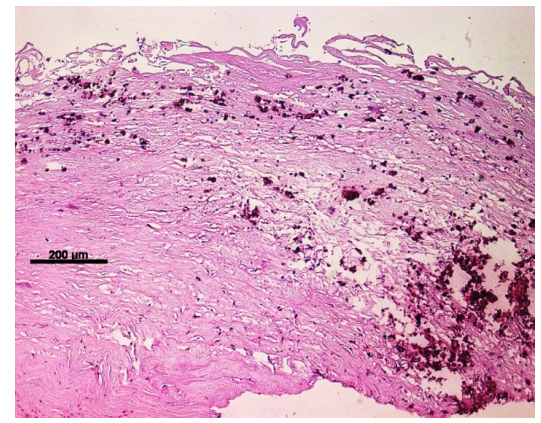

(a)

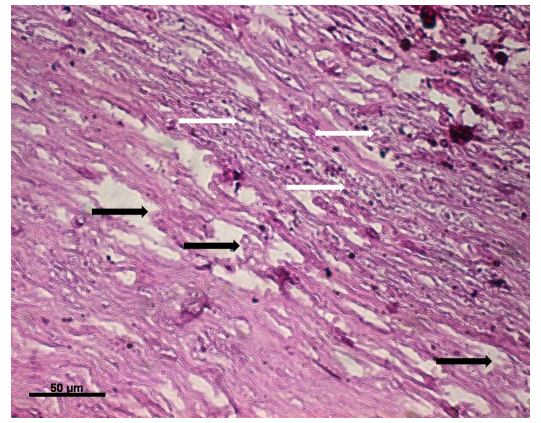

(b)

Figure 3: Cross-section of the arterial wall aneurysm (dome). (a) - the presence of large foci of calcification (bright black and purple blotches), as well as zones of calcification diffuse around the primary focus. (b) - microfragmentation of artery wall structures (white arrows). Fiber breaks and defects (black arrows) in the stroma of aneurysm wall. Stained with hematoxylin-eosin.

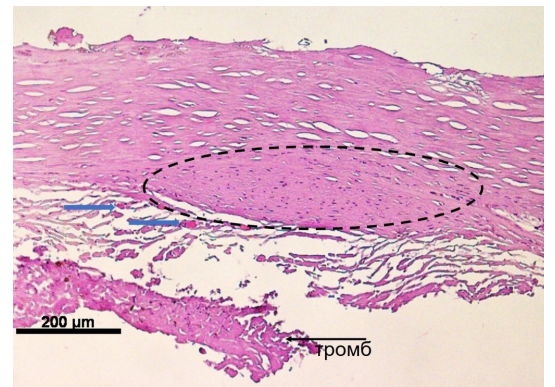

(a)

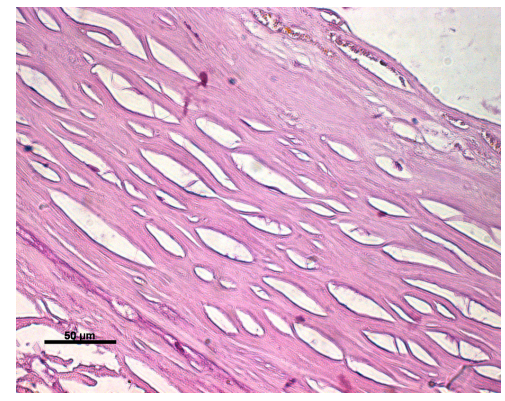

(b)

Figure 4: Cross-section of arterial wall aneurysm (neck): a) zone of intact cellularity (contour dotted) and microvessels (blue arrows); b) loss of fiber structure, aneurysm wall decellularization. Stained with hematoxylin-eosin.

\subsection{Fragment T. (neck).}

From the side of the intima, there is a fragment of the old parietal thrombus in the process of restructuring.,In the neck of the aneurysm, there is a lack of cell nuclei (hypocellular wall) in the stroma and fibers are destructured, with the separate zone on normal cellularity (4a).

At higher magnification, the destructurisation of the vascular wall (lack of layer in the structure of the vessel wall) and the initial stages of calcification are observed (Fig. 4b).

\section{Discussion}

This study is preliminary and the authors understand the limitations of the obtained results, since we have investigated only one patient using the described technique so far. During the 


\begin{tabular}{|c|c|c|}
\hline Attribute/Specimen & T. dome & T. neck \\
\hline Maximum strain & 1.50007 & 0.500467 \\
\hline Maximum stress & 0.279878 & 0.247911 \\
\hline Interfiber swelling & + & + \\
\hline Neovascular genesis present & + & + \\
\hline Calcification & Focal microcrystalline & $\begin{array}{l}\text { Subintimal foci + diffuse } \\
\text { microcrystalline }\end{array}$ \\
\hline
\end{tabular}

Table 2: Comparison of mechanical test and histological analysis.

course of the study we established a similaruty with the results of [1] in the part of reduced celluarity in the samples of unruptured aneurysms. As for the calcification, it was discovered in both specimens that points to insignificant change of this parameter in the direction from the neck of the aneurysm closer to the geometric center of its dome. However, the results of a mechanical experiment show that even minor changes in the histology of the samples can cause significant differences in the maximum strain.

According to the [13], this model of loss of cellularity in the wall of aneurysm of a vessel of the brain most of all corresponds to type C (loss of cellularity of the wall, lack of endothelium). We believe that the loss of smooth muscle cells and fibroblastic cells leads to ageing and the gradual destruction of the fibers of the connective tissue framework of the artery wall, which surely entails a change in the mechanical strength in this vascular zone. However, at this stage of research, we see only the consequences of the pathological process (the formation of the aneurysm) while the real reason for the launch of these negative changes is not completely clear.

An interesting fact is that when hemotoxylin-eosin hystology method is used, the general nature of tissue changes in the similar manner to the changes that occur in vascular atherosclerosis, despite the fundamental difference in the physiology of the processes, but for a more thorough comparison it is necessary to study a larger number of samples.

This study was partially supported by a grant from Russian Foundation for Basic Research grant No 19-48-540010. Also authors thank Russian Government grant No 14.W03.31.0002 for access to the Instron 5944 rupture machine unit.

\section{References}

[1] S. Morel, M.R. Diagbouga, N. Dupuy, E. Sutter, V. Braunersreuther, G. Pelli, M. Corniola, R. Gondar, M. Jägersberg, N. Isidor et al., Journal of Neuropathology \& Experimental Neurology 77, 555 (2018)

[2] D. O Wiebers, J. P Whisnant, J. Huston, I. Meissner, R. D Brown, D. G Piepgras, G. S Forbes, K. Thielen, D. Nichols, W. M O'Fallon et al., Lancet 362, 103 (2003)

[3] J.R. Cebral, X. Duan, B.J. Chung, C. Putman, K. Aziz, A.M. Robertson, American Journal of Neuroradiology 36, 1695 (2015)

[4] V. Costalat, M. Sanchez, B. Ambard, Thines, F.N. N Lonjon, H. Brunel, J. Lejeune, H. Dufour, P. Bouillot, J. Lhaldky et al., Journal of Biomechanics 44, 2685 (2011)

[5] H. Brunel, D. Ambard, H. Dufour, P.H. Roche, V. Costalat, F. Jourdan, Journal of Biomechanics 77, 76 (2018)

[6] A. Lipovka, K. Ovsyannikov, A. Dubovoy, D. Parshin, Journal of Physics: Conference Series (2018) 
[7] D. Parshin, A. Lipovka, A. Yunoshev, K. Ovsyannikov, A. Dubovoy, A. Chupakhin, Scientific reports (Accepted)

[8] A. Valencia, A. Contente, M. Ignat, J. Mura, E. Bravo, R. Rivera, J. Sordo, Journal of Mechanics in Medicine and Biology 15, 1550075 (2015)

[9] A.M. Robertson, X. Duan, K. Aziz, M.R. Hill, S.C. Watkins, J.R. Cebral, Annals of Biomedical Engineering 43, 1502 (2015)

[10] R. Gaul, D. Nolan, C. Lally, Journal of Biomechanics 81 (2018)

[11] N. Etminan, R. Dreier, B. Buchholz, K. Beseoglu, P. Bruckner, C. Matzenauer, J. Torner, R.J. Brown, H. Steiger, D. Hänggi et al., Stroke pp. 1757-63 (2014)

[12] D. Parshin, E.E. Tsibulskaya, A. Dubovoy, N. Maslov, AIP Conference Proceedings (2019)

[13] J Med Discov, E-Discovery, X. Duan, Journal of Medical Discovery 2, jmd17001 (2017) 